The following pages constitute the final, accepted and revised manuscript of the article:

Eklund, Mona and Bäckström, Martin

"A model of subjective quality of life for outpatients with schizophrenia and other psychoses.”

Qual Life Res. 2005 May;14(4):1157-68.

Publisher: Springer

Use of alternative location to go to the published version of the article requires journal subscription.

Alternative location: http://dx.doi.org/10.1007/s11136-004-2960-7 


\section{A Model of Subjective Quality of Life for Outpatients with Schizophrenia and other Psychoses}

Running head: Determinants of Quality of Life

Mona Eklund ${ }^{*}$, Professor, Ph.D., Lund University, Department of Clinical Neuroscience, Division of Occupational Therapy, PO Box 157, SE-221 00 Lund, Sweden.

Tel +4646 2221957

Fax +46462221959

E-mail: mona.eklund@med.lu.se

Martin Bäckström, Assistant Professor, Ph.D., Department of Psychology, Lund University, Lund, Sweden

\footnotetext{
${ }^{*}$ Corresponding author
} 


\section{Abstract}

This study investigated predictors of quality of life among persons with schizophrenia and other psychoses. On the basis of previous research, it was hypothesised that objective life circumstances, self-variables, psychopathology, activity level, satisfaction with daily activities, and satisfaction with medical care would be determinants of quality of life. 134 persons were investigated, and the analysis was based on Structural Equation Modelling (SEM). A model with two quality of life variables, General quality of life and Security, fitted the data best. A self-variable, Sense of self, and Satisfaction with daily activities, Psychopathology, and Satisfaction with medical care served as mediators and explained the variation in the quality of life variables. External to the mediators, and related to these, were Activity level, Educational level, and Independent living. In conclusion, a self-variable showed the strongest association with quality of life, but two aspects that should be feasible to influence in mental health care, daily activity and medical care, contributed to the subjects' self-rated quality of life. These results render a somewhat optimistic view on how to accomplish a better self-perceived quality of life among people with severe mental illness.

Key words: quality of life, schizophrenia, self psychology, psychopathology, socioeconomic factors, activities of daily living, medication. 


\section{Introduction}

This paper addresses quality of life as perceived by the individual, defined as wellbeing within a number of life areas, such as living situation, social relationships, and physical and mental health [1]. Numerous studies have tried to explain what determines subjective quality of life among people with mental illness [2-7], and recent research acknowledges that complex models with several factors are needed to give fair explanations. For example, Zissi and associates [8] explored a model that tried to explain variation in quality of life as being dependent on a complexity of relationships between objective indicators and quality of life mediators, such as a self-factor and autonomy. They found empirical support for a link between the mediators and subjective quality of life. No direct relationships were found between objective quality of life indicators and subjective quality of life but there were some relationships between objective indicators and the mediators. The most promising attempts to explain quality of life are considered to be those that are based on analysis of latent variables [9-10]. One such study found that autonomy contributed to quality of life, while diagnosis, social contacts, activity, and use of services did not [5]. A more recent study [11] showed that personality and self-variables were the most important contributors to self-rated quality of life, while psychopathology and objective indices could explain additional parts. They also found that quality of life was best explained by two constructs, one external aspect, composed by work and finances, and one internal, where health, security, and social relationships were important domains.

In a review of quality of life in severe mental illness, Holloway and Carson [12] suggested that personal characteristics, self-constructs, and clinical characteristics may be mediators, influencing how objective circumstances and subjective appraisal determine perceived quality of life. This was confirmed in a study by Eklund et al. [11]. Thus, research based on multi-factorial modelling and research overviews have come to some conclusions 
regarding factors that influence the quality of life of persons with severe mental illness. However, there are additional factors that so far have not been tried in multi-factorial models. Some years ago Awad and associates [13] proposed that compliance with medication and side-effects of medication were the most important explanations to quality of life among people with schizophrenia. Furthermore, a previous study from our own research group pointed to a strong relationship between satisfaction with daily activities in a broad sense and self-rated quality of life [2]. Also the performance of daily activities, the actual doing and people's degree of engagement in activities, seems to contribute to people’s quality of life [14-15]. This made us design a new study, in order to test a model that included the so far known and suggested factors from multi-factorial research but also comprised variables indicated to be of importance in correlational and qualitative studies. The summary of research above so far indicates that such a model should comprise personality factors, selfvariables, objective indices, psychopathology, daily activities, and medical care. However, although a previous study [11] showed that both personality factors and self-variables were important contributors to quality of life, they shared a considerable amount of variance. Thus, one might argue that self-variables alone would serve as a satisfactory estimate of personality and self. To the best of our knowledge, activity level, satisfaction with daily activities, and satisfaction with medical care have not been tested before in multi-factorial quality of life research. Knowing more about these variables is of importance for theoretical reasons, because they can contribute to the knowledge about self-rated quality of life, and for practical reasons, since they can be monitored by the health and social care system. Therefore, a special focus was put on them.

\section{Aim}

This study aimed at developing and testing a multi-factorial model for further clarifying selfrated quality of life. Based on previous research, it was hypothesised that self-rated quality of 
life in a sample composed by people with psychosis, mainly schizophrenia, could be explained by a model comprising self-variables, objective indices, psychopathology, activity level, satisfaction with daily activities, and satisfaction with medical care.

\section{Methods}

\section{Selection procedure}

Selection criteria were persistent mental illness ( $>$ two years of contact with mental health care) and an age of 20-55 years. Persons with co-morbidity of developmental delay or dementia, or who were too confused to participate, were excluded. In order to obtain sufficient statistical power two samples fitting the initial criteria but selected from two different units on partially different additional criteria were included. At the first unit, all patients diagnosed with schizophrenia or related disorders and with at least one visit to the unit during the past one-year period were asked to participate. This procedure identified 119 individuals. A dropout of altogether 45 individuals resulted in a participation rate of $62 \%$. There were no differences between the dropouts and the participants regarding sex $(p=0.545)$, age ( $\mathrm{p}=0.457)$, or diagnostic subgroups of patients $(\mathrm{p}=.192)$. The second subsample was involved in a project that included comparisons between patients with competitive work or studies, patients with other forms of regular and structured daily activities, and patients with no regular and structured daily activity [16]. For that project, all patients at an outpatient clinic for people with persistent mental illness fitting the initial criteria were first grouped in three strata representing these forms of daily activities. Eligible subjects were then randomly selected from these strata. In all, 174 patients were asked to participate, 103 of which agreed, giving a participation rate of $60 \%$. A dropout analysis showed that the participants were somewhat younger than those who declined participation (39/42 years, $\mathrm{p}=0.021)$ and contained fewer women $(45 / 61 \%, \mathrm{p}=0.04)$. There was no difference between participants and 
dropouts as to diagnostic subgroups. For the present study those with a diagnosis of schizophrenia or some other type of psychosis, 60 patients, were selected. For both subsamples, the psychiatrists in charge also diagnosed the patients, in the first sample according to DSM-IV [17] and in the second according to ICD-10 [18]. That different diagnostic taxonomies were used depends on different clinical praxis in the two units. A research ethics committee approved both projects on which this study was based, and the principle of informed consent was used.

\section{Subjects}

The subjects of the sample as a whole had a mean age of 41.5 years $(\mathrm{SD}=8)$, ranging from 20 to 55 years. There were 51 women (38\%) and 83 men (62\%). Most respondents were native Swedes (86\%), lived alone (67\%) and had no children (75\%). The most common type of living accommodation was an apartment, and 82\% lived independently, inasmuch as that they had an apartment of their own or, in a few cases, a house. Most of the subjects currently had a sick or disability pension. The predominant main diagnosis was schizophrenia $(n=103,77 \%)$. Common diagnostic subgroups were paranoid form $(n=54)$, hebephrenic form $(n=17)$, and undifferentiated form $(\mathrm{n}=15)$. Seventeen persons had a schizophrenia-related disorder, whereof ten had a schizoaffective disorder. Fourteen had some other form of psychosis, the most common one being acute and transient psychotic disorder $(n=8)$. At the time of data collection all subjects were in regular contact with psychiatric services.

\section{Instruments}

\section{The quality of life}

As a measure of quality of life the Manchester Short Assessment of Quality of Life (MANSA) [19] was used. It is a short version of the Lancashire Quality of Life Profile (LQLP) [20], which has been translated into Swedish and found to be psychometrically sound 
[21]. Good agreement has been shown between the MANSA and the LQLP [19]. MANSA is administered as a structured interview and includes the individual's subjective rating of their general life satisfaction as well as satisfaction concerning different quality of life domains: work, finances, social relations, leisure, living situation, safety, family relations, sexual relations, and health. The ratings are made on a seven-point scale and the mean ratings from the different domains form an overall quality of life score. MANSA also includes a number of questions regarding objective indices pertaining to the domains, such as type of accommodation, earnings, etc. A Cronbach’s alpha analysis based on the present sample resulted in a coefficient of 0.81 , indicating good internal consistency.

\section{Objective life circumstances}

To reflect this area an interview-based questionnaire devised for this study was used, and in addition the objective questions regarding the life domains registered in the MANSA were used.

\section{Psychopathology}

Psychiatric symptoms were assessed by means of the Brief Psychiatric Rating Scale (BPRS) [22]. It consists of 18 items and allows for analysis into positive, negative, and depressive symptoms and general psychopathology. Good inter-observer and intra-observer reliability has been demonstrated, in this original 18-items version as well as in an extended version [2324]. The Cronbach's alpha value for the present sample was 0.79 .

Global Assessment of Functioning (GAF) [17] was employed to estimate global psychosocial functioning. It forms a single rating on a 100-point scale, where 100 indicates not only absence of pathology but also positive mental health, and has acceptable reliability and validity [25-26]. 


\section{Self-variables}

The Sense of Coherence (SOC) scale [27], which indicates how well a person manages stress and stays healthy even in trying circumstances and traumatic experiences, was used in this study as a self-related aspect. The respondents rate questions reflecting the constructs comprehensibility, manageability, and meaningfulness on a 7-point scale with two anchoring responses (e.g., never and very often). The instrument has proven to be valid and reliable [2829]. A short version with 13 items, shown to have the same properties as the original 29-item scale [30], was used. A Cronbach’s alpha analysis based on the present sample resulted in a coefficient of 0.87 .

The self-esteem scale, originally developed by Rosenberg [31], was used to measure another self-aspect. It is based on 10 items, 5 that tap positive judgements towards the self and 5 that are formulated as negative attitudes towards the self. Self-esteem is expressed as the difference between positive and negative self-attitudes. The scale has been shown to have acceptable internal consistency [20], and alpha values for the present study were 0.77 for the positive items and 0.70 for the negative.

Perceived control was measured by means of two constructs-mastery and locus of control. Mastery [32] is defined as "the extent to which people see themselves as being in control of the forces that importantly affect their lives” (p. 340). The scale has been found empirically distinct and to have good internal consistency [33]. The Locus of Control (LOC) scale, constructed by Rotter [34], refers to whether an individual perceived reinforcements to be a function of his own actions (internal [I] control) or externally determined (external [E] control). The present study used a variant of the instrument developed for Sweden [35]. The LOC scale has a fair internal consistency and test-retest reliability [34]. The Cronbach alphas for the present sample were 0.81 for the Mastery scale and 0.69 for Locus of Control. 


\section{Daily activities}

Performance of and satisfaction with daily activities were assessed by means of an interviewbased questionnaire developed for a previous study by this research group [2]. It has good internal consistency and satisfactory predictive and discriminant validity [36]. The alpha coefficient for the present sample was 0.80 . The instrument consists of seven items regarding the activity areas of work (four items: being employed, being in work the past month, participating in work rehabilitation, and participation in community-based activity centres), leisure activities (two items: organised activities and individually enjoyed leisure), and domestic tasks (one item). Each item consists of a two-part question. The first part queries whether or not the subject performs the targeted kind of activity at the moment. The response format is yes/no. The second asks the subject to rate his/her satisfaction on a seven-point scale, i.e., a person who performs domestic tasks rates his or her satisfaction with being involved in such activities, and a subject without involvement in domestic tasks rates that condition. Two activity indexes can be calculated from this scale - one that reflects the level of engagement in daily activities and is based on the first part of each question. A maximum of seven points can be obtained - one point for each activity that is presently performed. The second index concerns satisfaction with daily activities and is expressed as a mean of the satisfaction ratings. Since satisfaction with work and leisure activities are included in the quality of life instrument as well, only satisfaction with work rehabilitation, community-based activity centres, domestic tasks, and self-care were allowed to form the satisfaction with activity aspect in this study. Since the mere participation in activities is not reflected in the quality of life instrument used, all activity areas were included in the activity level aspect.

\section{Satisfaction with medical care}

The interview questionnaire tapping objective life circumstances also included questions on satisfaction with care. Two aspects were chosen for this study, namely satisfaction with 
medication and satisfaction with contacts with the psychiatrist. They were set to form an index of satisfaction with medical care. Alpha for these two items was 0.60.

\section{Data analysis}

This study was based on Structural Equation Modelling (SEM), where the general idea is to create different hypothetical models based on competing theories within a specific field of research and investigate which one of them fits the data best. The first step encompassed a measurement model of self-rated quality of life. Thereafter, in a second step a number of pathanalyses were conducted to model the relations between the included variables. In all analyses we used direct measures of the constructs and refrained from using latent variables because of the rather low number of subjects.

On the basis of findings from previous investigations of quality of life, as accounted for in the introductory part, six different areas of predictors were hypothesised to account for the variation in quality of life: Objective life conditions (further specified below), Psychopathology, Sense of self, Activity level, Satisfaction with daily activities, and Satisfaction with medical care. Psychopathology comprised the GAF scores and the BPRS subscales positive, negative, depressive, and general symptoms. Included in, Sense of self, were the variables mastery, locus of control, sense of coherence, and self-esteem. The objective life conditions were, independent living in one’s own flat or house, level of education, having friends, having children, being married, living with someone (spouse, parents, children or friend), sex and age.

The software used for the structural equation modelling was LISREL 8.54 [37]. The STREAMS program [38] was used as an aid to simplify the process of defining the models and generating start values. The fit between the data and a model was estimated by $\chi^{2}$, by the fit index Root Mean Square Error of Approximation (RMSEA), and the Comparative Fit Index (CFI), and the Parsimony Normed Fit Index (PNFI). The RMSEA estimates the lack of fit in a 
model compared to a perfect model; low values, below 0.05 , indicate good fit. The CFI estimates fit, and a value above 0.95 indicates good fit. This measure is not as sensitive to sample size as other similar indices. The PNFI takes into account the parsimony of the mode and should be as high as possible. In some cases we compared models, and accordingly we use $\Delta \chi^{2}$, i.e., the difference in $\chi^{2}$ between models. In Table 3 a number of other well-known indexes [37] are shown as well. All presented statistics were estimated with the maximum likelihood method.

\section{Results}

The first estimations concerned a measurement model and tested if the dependent variables, the different domains of subjective quality of life, consisted of one or more factors. Table 1 displays loadings regarding two different estimations related to this question, and Table 2 shows correlations among all the variables used in this study.

\section{TABLE 1}

\section{TABLE 2}

A model with a single factor had a rather good fit (Model MeasQOL1 in Table 3), but the significant chi-square and some of the fit indexes suggested that a better fit was possible. The subscale finance had a conspicuously weak negative loading to this single factor (Table 1). It was decided to create a new factor based on results from an exploratory factor analysis using principal components and varimax rotation. The exploratory factor analysis suggested that the quality of life measures used here could be summarised by two factors. The first factor, related to all subscales except economy, was labelled General quality of life. The second factor seemed to measure a contrast between satisfaction with finances and four other quality of life subscales, friends, safety, living situation, and family relations. With respect to this second factor, further on referred to as Security, satisfaction with finances had a negative 
loading while the other four subscales had positive loadings. This two-factor model had a significantly better fit (Model MeasQOL2 in Tables 1 and 3; $\Delta \chi^{2}=24.95 ; \Delta d f=5 ; p<0.05$ ) compared to the single factor model. All fit indexes except PNFI indicated better fit. Freeing covariation between the factors did not result in a better fit $\left(\Delta \chi^{2}=0.69 ; \Delta d f=-1 ; p>0.05\right)$, suggesting the two factors to be more or less uncorrelated. The modification indexes of the two-factor model suggested the subscales physical health and psychological health to be correlated $\left(\Delta \chi^{2}=12.13 ; \Delta d f=-1 ; p<0.05\right)$. Apart from this, it seems that a two-factor model was a viable alternative to a one-factor model in explaining the covariation between the quality of life subscales.

\section{Path models}

In the first set of path models there were seven summarised variables, two dependent, measuring the aspects of quality of life, and five independent measuring Sense of self, Pathology, Satisfaction with medical care, Satisfaction with daily activities, and Activity level. Among the variables measuring objective life conditions, three were included based on their significant correlations to the quality of life variables. These were, having a close friend or not (Friendship), Independent living in one's own flat or house, and Educational level. Five variables were excluded since they did not correlate with quality of life, and these were, being married or not, having children or not, living with someone or not, subject sex, and age. The quality of life variables were created by weighing the subscales according to the coefficients obtained from the confirmatory factor analysis above.

The first model (Model 1) had paths from all independent variables to all dependent variables. This model did not have a very good fit, $\chi^{2}(29, n=134)=237.39 ; p<0.001$. The $C F I$ was 0.70 and the RMSEA was 0.29. If the non-significant paths were deleted (Model 2), the RMSEA decreased to 0.23 . There was considerable covariation between the independent variables, and if this covariation was included, the model (Model 3) became considerably 
better with $\chi^{2}(9, n=134)=6.19$, having a $\Delta \chi^{2}$ of 237.24 with $\Delta d f=25$, clearly a significant improvement. The CFI for this model was 1.0 and the RMSEA was 0.00 . This was not a very parsimonious model indicated by the decrease in the adjusted indexes (e.g. PNFI fell from 0.55 to 0.20$)$.

Model 3 was almost saturated, with only nine degrees of freedom, eight of them stemming from the excluded non-significant paths to quality of life. Figure 1 displays this model and all the significant path coefficients. The strongest direct effects on General quality of life were from Satisfaction with daily activities (0.33), Sense of self (0.44), Satisfaction with medical care (0.13), and Psychopathology (-0.22). With respect to the second quality of life factor, Security, the following coefficients were found: Satisfaction with daily activities (0.31), Satisfaction with medical care (0.12), Pathology (0.24), and Educational level (0.19). All other correlations were insignificant.

The next step was a more interesting path model, where Sense of self, Psychopathology, Satisfaction with medical care, and Satisfaction with daily activities were defined as mediators. Paths smaller than 0.10 were deleted, resulting in Model 4 (Table3). Compared to Model 1 above, this model takes some of the covariation between the independent variables into account. There were significant coefficients to Sense of self from Educational level (0.13) and Activity level (0.34), to Pathology from Independent living (-0.35), Educational level (0.16) and Activity level (-0.28), to Satisfaction with medical treatment from Educational level (0.25) and Activity level (0.20), and to Satisfaction with daily activities from Activity level (0.34). The fit for this model was far from good, with $\chi^{2}(28, n=134)=127.26 ; p>0.05$. The $C F I$ was 0.83 and the RMSEA was 0.17 (Table 3). The covariation between the mediators was accounted for in Model 5, resulting in a better fit $\left(\Delta \chi^{2}=-68.65 ; \Delta d f=-6 ; p<0.001\right)$.

One focus of this study was the contribution of the subjects' satisfaction with their medical treatment and with their daily activities. These two variables' relation to quality of 
life was tested separately. First, Satisfaction with daily activities was excluded and then reintroduced in Model 5, and the results showed that Satisfaction with daily activities contributed to quality of life $\left(\Delta \chi^{2}=-101.78 ; \Delta d f=2 ; p<0.001\right)$. Next, Satisfaction with medical care was tested in the same way. The analysis showed that Satisfaction with medical care significantly contributed to quality of life as well $\left(\Delta \chi^{2}=-8.22 ; \Delta d f=2 ; p<0.02\right)$.

In the last step (Model 6) coefficients were set free for covariation between all the independent variables not defined as mediators (Activity level, Educational level, Independent living, and Friendship). In addition the covariation between the quality of life variables was freed, and together this increased the fit considerably $\chi^{2}(16, n=134)=9.09 ; p=0.045$. CFI was 0.99 for this model and RMSEA was 0.000. The last model (Model 6) is displayed in Figure 2. The coefficients between the mediators and the dependent variables for this model were almost exactly the same as for Model 3. Regarding the other independent variables, Activity level was related to all the mediators, with the largest coefficient to Satisfaction with daily activities. Educational level was related to Satisfaction with medical care, Pathology, and the Sense of self, but had in addition a direct path to the second quality of life variable, Security. Moreover, Independent living was negatively related to Pathology and somewhat positively associated with Satisfaction with medical care. Friendship was not related to any of the mediators and did not significantly contribute to quality of life in this final model.

\section{Discussion}

This study aimed at estimating factors of importance to quality of life in a rather large group of subjects diagnosed with severe mental illness. It could confirm that a self-factor seems to be an important aspect of self-rated quality of life. It also confirmed that degree of psychopathology and certain objective life conditions, in this case independent living and level of education, can explain additional proportions of the variation in quality of life. Moreover, the study showed that some variables, not tested previously in multi-factorial 
quality of life research, were significant contributors. These variables concern medical care and daily activities and will be discussed in some more detail.

In line with previous research by Awad et al. [13], our study showed that a factor related to the psychotropic treatment of the patient, Satisfaction with medical care was of importance for the quality of life. Even though this study did not use exactly the same variables as the study by Awad and associates, both investigations tap the same area - the medical care. However, the present study goes beyond the scope of Awad's study in that medical care was framed in a multi-factorial model. As a result, the contribution of medical care in the present study, although significant, was much less prominent than in the Awad study. The other mediators - Sense of self, Satisfaction with daily activities, and Psychopathology - all contributed more to the variation in self-rated quality of life. This accords with Ritsner and associates [39], who found that medication side-effects influenced subjective quality of life less than other clinical and psychosocial factors. A note of warning regarding the comparison of coefficient sizes is that variation in reliability could partly explain these differences. With respect to Satisfaction with medical care it is not possible to accurately estimate the reliability due to the low number of items in this scale (only two). Thus, Satisfaction with medical care was positively related with both quality of life factors, which is interesting from a clinical point of view, since this factor should be feasible to influence, e.g., by careful monitoring of the psychotropic medication and efforts to improve the therapeutic relationship between patients and staff. Similarly, activity aspects have shown to correlate highly with self-rated quality of life [2], but have not previously been analysed in a multi-factorial model. Satisfaction with daily activities was set as a mediator, together with other variables based on ratings, while Activity level was set among the objective life conditions, since it reflects an actual circumstance - the number of activity areas an individual is engaged in. The contribution of Satisfaction with daily activities to the quality of life factors 
was less than in the previous study by Eklund et al., but was the second most important contributor to both of the quality of life factors in the present study, next to Sense of self.

Educational level was the only unmediated variable with direct influence on a quality of life factor, namely Security. All other independent variables in the model were related to the mediators, but not to quality of life when the mediators were accounted for. Activity level showed to be related to all mediators, and contributed most among the unmediated variables to both Sense of self and Satisfaction with activities. Educational level contributed most to Satisfaction with medical care, but was not related to Satisfaction with activities. Independent living was closest associated with Psychopathology. Besides that, Independent living was only weakly associated with Satisfaction with medical care. Thus, among the exogenous variables, Activity level had the strongest relation to the mediators, emphasising that daily activities are of importance for satisfaction variables and therefore should be carefully addressed in mental health care.

Quality of life tends to be a complex phenomenon. Just as in previous studies by our research group [11] and by Meijer and associates [40], the results from the present study indicated that quality of life was composed by two factors, General quality of life and Security. However, the two-factor solutions arrived at were somewhat different in our two studies. While one inner, personal aspect and one more materialistic, outer aspect were found in the study by Eklund et al. [10], the present study pointed to one general and one securityoriented factor. Thus, there seems to be some general consistency in findings over different studies, but differences in particularities, a dilemma that constitutes a challenge for future theory-generating research into quality of life.

General quality of life ought to be the most important one of the two quality of life factors, formed largely by Sense of self and Satisfaction with daily activities. Any change in these variables should affect general quality of life. The self is composed by a combination of 
basic personality variables, the objective personal history, adaptation, and external situational influences [41]. Thus, although not so stable as personality variables, the self is not so easily accessible for intervention and change. Therefore, in order to improve quality of life in general, Satisfaction with daily activities seems to be an important parameter, in turn influenced by the actual engagement in activities.

The trustworthiness of our final model (Figure 2) should be addressed. The variables based on ratings, either self-ratings or interviewer ratings were set as mediators, whereas variables pertaining to actual circumstances were assumed to be related to quality of life through the mediators. However, setting the suggested variables as mediators of quality of life was not suggested by the data itself, it was based on the argument that aspects of self, pathology, and satisfaction with different important areas in life precede and determine quality of life. Logically, the relationships from Sense of self and Pathology to quality of life should be in the direction suggested in our model. For the other mediators it could be the other way around, however, that the quality of life experienced determines the Satisfaction with daily activities and Satisfaction with medical care. That the causal relationship goes from the selected mediators and exogenous variables to quality of life is a theoretical assumption, but was supported by our final model. The theoretical rationale for this model based on mediators may be defended from another point of view as well. There is no research evidence for the assumption that a person would be satisfied with an area of life only based on objective life circumstances [10, 42-43]. It is how these life circumstances are appraised and valued that is decisive for the experienced quality of life.

One of the obvious shortcomings in the present research is the lack of data on some important aspects of the respondents’ illness, such as psychotropic medication, its sideeffects, and duration of illness, which could have served as additional exogenous variables, influencing the mediators and quality of life. Similarly, personality, shown to be of 
importance in quality of life research [3-4, 7], was not specifically addressed, since the covariation with a self-factor was substantial in a previous study [11]. For practical and economical reasons, it is very difficult to include all measures in one single model, and this is especially true for exogenous factors.

A model based on latent variables was not presented in this study because of the small number of subjects in relation to the number of coefficients. However, we actually explored a model built on latent variables as well, and that model did not differ from our final model, except for that some the coefficients were higher. It is well known that the size of the coefficients are uncertain when there is lack of reliability in the measurements, something that could have been solved if a model with latent variables had been used.

Our model suggests that some of the variables mediated other variables, and this has been argued for above. The intention was to create a model that makes the interrelations between variables more explicit. An obvious possibility, not utilised in the present study, would be the inclusion of moderators. Among others, Baron and Kenny [44] have defined the difference between mediators and moderators. The variables suggested as mediators in our study clearly acts as such; together they either function as total or partial mediators of the exogenous variables. There has been no attempt to include moderators, however. That would have made the model immense, especially if all possible interactions among the independent variables should be tested.

A real methodological challenge for this kind of research is the fact that predictors of quality of life, both on the level of measurement and conceptually, are highly correlated and interrelated. This was indicated by the increase in fit when the correlations between independent variables were included in the models. It is tempting to create even simpler models where such covariations are subsumed under more global concepts than used here. It is important, however, that the concepts used in a model like this are clinically relevant, that 
they are not estranged from reality. To achieve this it is sometimes necessary to include factors in the model that are interdependent. The choice of predictors must be evaluated in relation to how useful they are to people who work with mentally ill persons. The addition of the mediators Satisfaction with medical care and Satisfaction with daily activities to our previously presented model [11] could then be defended, arguing that they are clinically very important, warranting inclusion in our new model even if they partly measure concepts already included in the previous model.

In general, people with schizophrenia tend to rate their quality of life higher than, e.g., people with mood disorders [45-46], and it is likely that the pattern of predictors might differ between diagnostic groups. This was recently indicated by Ritsner et al. [47]. Consequently, it is possible that some of the predictors in the present study, e.g. medication, might be of different significance for patients suffering from schizophrenia and other psychoses as compared to other forms of mental illness. Therefore, diagnosis is a variable that should be considered in this type of research. The validity of the diagnoses could be questioned in this study, since different diagnostic taxonomies were used for the two samples. Preferably, the DSM-IV system should be used for research purposes, but this was not feasible in one of the units. However, the ICD-10 is frequently used the whole world over to make clinical diagnoses on which treatment is based, and should be valid enough for the purpose of this study, which did not require any division into sub-diagnoses.

The model resulting from this study must be regarded as specific for people with schizophrenia and related disorders, and it shows similarities with results from other studies on this group $[8,11-12,48]$. Since research has shown that the predictors of subjective quality of life tend to differ between patients with schizophrenia and, e.g., mood disorders [47], future research should develop predictor models for groups with other psychiatric diagnoses as well, such as mood and anxiety disorders. 


\section{Conclusion}

This study revealed that daily activity and medical care contributed to self-rated quality of life among severely mentally ill patients, besides previously well-known predictors such as a selfvariable and psychopathology. This adds to the theoretical understanding of self-rated quality of life and gives some implications for clinical practice, since both daily activity and medical care are aspects that should be feasible to influence in mental health care. Even if the not so easily manipulated self-factor was the most influential mediator, the results render a somewhat optimistic view on how to accomplish a better self-perceived quality of life in the target group. Also, the results suggest that different aspects of daily activities and medical care should be carefully addressed when assessing the quality of life in the target group. 


\section{References}

1. Post MWM, de Witte LP, Schrijvers AJP. Quality of life and the ICIDH: Towards an integrated conceptual model for rehabilitation outcomes research. Clin Rehabil 1999; 13: 5-15.

2. Eklund M, Hansson L, Bejerholm U. Relationships between satisfaction with occupational factors and health-related variables in schizophrenia outpatients. Soc Psychiatr Epidemiol 2001; 36: 79-85.

3. Hansson L, Eklund M, Bengtsson-Tops A. The relationship of personality dimensions as measured by the TCI and quality of life in individuals with schizophrenia or schizoaffective disorder living in the community. Qual. Life Res. 2001; 10: 133-139.

4. Kentros MK, Terkelsen K, Hull J, Smith TE, Goodman M. The relationship between personality and quality of life in persons with schizoaffective disorder and schizophrenia. Qual Life Res 1997; 6: 118-122.

5. Mercier C, King S. A latent variable causal model of the quality of life and community tenure of psychotic patients. Acta Psychiatr Scand 1994; 89: 72-77.

6. Norman RMG, Malla AK, McLean T, Voruganti LPN, Cortese L, McIntosh E, Cheng S, Rickwood A. The relationships of symptoms and level of functioning in schizophrenia to general wellbeing and the quality of life scale. Acta Psychiatr Scand 2000; 102: 303-309.

7. Ritsner M, Farkas H, Gibel A. Satisfaction with quality of life varies with temperament types of patients with schizophrenia. J Nerv Ment Dis 2003; 191:668-674.

8. Zissi A, Barry MM, Cochrane R. A mediational model of quality of life for individuals with severe mental health problems. Psychol Med 1998; 28: 1221-1230. 
9. Ruggeri M, Warner R, Bisoffi G, Fontecedro L. Subjective and objective dimensions of quality of life in psychiatric patients: A factor analytical approach. Br J Psychiatry 2001; 178: $268-275$.

10. Ruggeri M, Gater R, Bisoffi G, Barbui C, Tansella M. Determinants of subjective quality of life in patients attending community-based mental health services. The South-Verona Outcome Project 5. Acta Psychiatr Scand 2002; 105: 131-140.

11. Eklund M, Bäckström M, Hansson L. Personality and self-variables important determinants of subjective quality of life in schizophrenia outpatients. Acta Psychiatr Scand 2003; 108: 134-143.

12. Holloway F, Carson J. Quality of life in severe mental illness. Int Rev Psychiatr 2002; 14: 175-184.

13. Awad AG, Voruganti LNP, Helsegrave RJ. A conceptual model of quality of life in schizophrenia: Description and preliminary clinical validation. Qual Life Res 1997; 6: 2126

14. Kelly S, McKenna H, Parahoo K. The relationship between involvement in activities and quality of life for people with severe and enduring mental illness. J Psychiatr Ment Health Nurs 2001; 8: 139-146.

15. Wilcock AA, van der Arend H, Darling K, Scholz J, Siddall R, Snigg C, Stephens J. An exploratory study of people's perceptions and experiences of wellbeing. Br J Occup Ther 1998; 61: 75-82

16. Eklund M, Hansson L, Ahlqvist C. The importance of work as compared to other forms of daily occupations for wellbeing and functioning among persons with long-term mental illness. Community Ment. Health J. (in press). 
17. American Psychiatric Association (APA). Diagnostic and Statistical Manual of Mental Disorders, 4th edn (DSM-IV). Washington, DC: APA, 1994.

18. World Health Organization. The ICD-10 Classification of Mental and Behavioural Disorders. Geneva: World Health Organization, 1993.

19. Priebe S, Huxley P, Knight S, Evans S. Application and results of the Manchester Short Assessment of Quality of Life (MANSA). Int J Soc Psychiatry 1999; 45: 7-12.

20. Oliver J, Huxley P, Bridges K, Mohamad H. Quality of Life and Mental Health Services. London: Routledge, 1996.

21. Hansson L, Svensson B, Björkman T. Quality of life of the mentally ill. Reliability of the Swedish version of the Lancashire Quality of Life Profile. Eur. Psychiatry 1998; 13: 231234.

22. Overall JE, Gorham DR. The Brief Psychiatric Rating Scale. Psychol. Rep 1962; 10: 799812.

23. Andersen J, Larsen JK, Schultz V, Nielsen BM, Kørner A, Behnke K, Munk-Andersen E, Butler B, Allerup P, Bech P. The Brief Psychiatric Rating Scale. Dimension of schizophrenia—reliability and construct validity. Psychopathology 1989; 22: 168-176.

24. Hafkenschied A. Reliability of a standardized and expanded Brief Psychiatric Rating Scale: A replication study. Acta Psychiatr Scand 1993; 66: 305-310.

25. Endicott J, Spitzer RL, Fleiss JL, Cohen J. The Global Assessment Scale. A procedure for measuring overall severity of psychiatric disturbance. Arch Gen Psychiatry 1976; 33: 766-771.

26. Friis S, Melle I, Opjordsmoen S, Retterstøl N. Global Assessment Scale and HealthSickness Rating Scale: Problems in comparing the global functioning scores across investigations. Psychother Res 1993; 3: 105-114. 
27. Antonovsky A. The structure and properties of the sense of coherence scale. Soc. Sci. Med 1993; 36: 725-733.

28. Bengtsson-Tops A, Hansson L. The validity of Antonovsky’s sense of coherence concept in a sample of individuals with schizophrenia living in the community. J Adv Nurs 2001; 33: 432-438.

29. Langius A, Björvell H, Antonovsky A. The sense of coherence concept and its relation to personality traits in Swedish samples. Scand J Car Sci 1992; 6: 165-171.

30. Langius A, Björvell H. Coping ability and functional status in a Swedish population sample. Scand J Car Sci 1993; 7: 3-10.

31. Rosenberg, M. Society and the Adolescent Self Image. Princeton, NJ: Princeton University Press, 1965.

32. Pearlin LI, Menaghan EG, Lieberman MA, Mullan JT. The stress process. J. Health Soc. Behav 1981; 22: 337-356.

33. Marshall GN, Lang EL. Optimism, self-mastery, and symptoms of depression in women professionals. J. Pers. Soc. Psychol. 1990; 59: 132-139.

34. Rotter JB. Generalized expectancies for internal versus external control of reinforcements. Psychological Monographs: general Applications 1966; 80: 1-28.

35. Eisemann M, Perris C, Palm U, Palm A, Perris H. LOC-presentation of a Swedish instrument for assessing locus of control. In: Perris C, Eisemann M, eds. Cognitive Psychotherapy—an Update. Umeå, Sweden: Department of Psychiatry, University of Umeå, 1988: 45-48.

36. Eklund M. Satisfaction with Daily Occupations - a tool for client evaluation in mental health care. Scand J Occup Ther; in press. 
37. Jöreskog KG, Sörbom D. LISREL 8: User’s Reference Guide. Chicago: Scientific Software International, 1993.

38. Gustafsson J-E, Ståhl PA. STREAMS 2.50.3: Structural Equation Modeling Made Simple. Mölndal, Sweden: MultivariateWare HB, 2000.

39. Ritsner M, Ponizovsky A, Endicott J, Nechamkin Y, Rauchverger B, Silver H, Modai I.. The impact of side-effects of antipsychotic agents on life satisfaction of schizophrenia patients: a naturalistic study. Eur Neuropsychopharmacol 2002; 12: 31-38.

40. Meijer CJ, Schene AH, Koeter MWJ. Quality of life in schizophrenia measured by the MOS SF-36 and the Lancashire Quality of Life Profile: A comparison. Acta. Psychiatr. Scand 2002; 105: 293-300.

41. McCrae RR, Costa PT Jr. A five-factor theory of personality. In: Pervin L, John OP, eds. Handbook of Personality: Theory and Research, 2nd edn. New York: Guilford, 1999: 139-153.

42. Gaite L, Vázquez-Barquero JL, Borra C, Ballesteros J, Schene A, Welcher B, Thornicroft G, Kuehner C. The Epsilon Study Group. Quality of life in patients with schizophrenia in five European countries: The Epsilon study. Acta. Psychiatr. Scand 2002; 105: 283-292.

43. UK700 Group. Predictors of quality of life in people with severe mental illness. Br J Psychiatry 1999; 175: 426-432.

44. Baron RM, Kenny DA. The moderator-mediator variable distinction in social psychological research: Conceptual, strategic and statistical considerations. J Pers Soc Psychol 1986; 51: 1173-1182.

45. Koivumaa-Honkanen HT, Konkanen R, Antikainen R, Hintikka J, Viinamaki H. Selfrated life satisfaction and treatment factors in patients with schizophrenia, major depression and anxiety disorder. Acta Psychiatr Scand 1999; 99: 377-384. 
46. Rudolf H, Priebe S. Subjective quality of life in female in-patients with depression in a longitudinal study. Int J Soc Psychiatry 1999; 45: 238-246.

47. Ritsner M, Kurs R, Gibel A, Hirschman S, Shinkarenko E, Ratner Y. Predictors of quality of life in major psychoses: a naturalistic follow-up study. J Clin Psychiatry 2003; 64: 30815.

48. Bechdolf A, Klosterkötter J, Hambrecht M, Knost B, Kuntermann C, Schiller S, Pukrop R. Determinants of subjective quality of life in post acute patients with schizophrenia. Eur Arch Psychiatry Clin Neurosci 2003; 253: 228-235. 


\section{Figure legends:}

Figure 1. Model 3 with coefficients - all independent variables free to co-vary.

Figure 2. Model 6 with coefficients - Psychopathology, Satisfaction with medical care, Satisfaction with daily activities, and Sense of self mediate quality of life; co-variation free between mediators and predictors, respectively. 
Table 1. Loadings related to the measurement models of the quality of life.

\begin{tabular}{l|ccc}
\hline & Measurement model 1 & \multicolumn{2}{c}{ Measurement model 2 } \\
\hline Quality of life items & QOLMeas 1 & QOLMeas2:1 & QOLMeas2:2 \\
Work & 0.27 & 0.26 & \\
Economy & -0.14 & & -0.32 \\
Friends & 0.45 & 0.34 & 0.26 \\
Leisure & 0.61 & 0.36 & \\
Accommodation & 0.64 & 0.47 & 0.43 \\
Safety & 0.58 & 0.38 & 0.59 \\
Physical health & 0.67 & 0.74 & \\
Psychological health & 0.74 & 0.79 & \\
Family relations & 0.53 & 0.40 & 0.32 \\
\hline
\end{tabular}

Note: QOLMeas2:1 is later on denoted as General quality of life (Qol G) and QOLMeas2:2 as Security, the safety aspect of quality of life (Qol S). 
Table 2. Correlations among all variables

\begin{tabular}{|l|rrrrrrrrrrrrr}
\hline & \multicolumn{1}{l|}{ Sex } & 1 & 2 & 3 & 4 & 5 & 6 & 7 & 8 & 9 & 10 & 11 & 12 \\
\hline Age (1) & -0.08 & & & & & & & & & & & & \\
Married (2) & 0.11 & -0.03 & & & & & & & & & & \\
Living (3) & -0.07 & -0.01 & -0.42 & & & & & & & & & \\
Friends (4) & 0.13 & -0.16 & 0.11 & -0.08 & & & & & & & & \\
Living (5) & 0.05 & -0.16 & 0.09 & 0.21 & 0.26 & & & & & & & \\
Education (6) & 0.03 & -0.09 & 0.15 & -0.06 & 0.20 & 0.31 & & & & & & \\
Sat med (7) & 0.02 & 0.08 & 0.08 & -0.07 & 0.19 & 0.25 & 0.34 & & & & & \\
Act level (8) & 0.03 & -0.05 & 0.01 & -0.05 & 0.26 & 0.30 & 0.31 & 0.31 & & & & & \\
Self (9) & -0.04 & 0.06 & -0.11 & -0.04 & 0.15 & 0.12 & 0.22 & 0.41 & 0.32 & & & & \\
Sat act (10) & 0.03 & 0.16 & 0.13 & 0.00 & 0.19 & 0.18 & 0.15 & 0.37 & 0.38 & 0.27 & & & \\
Ps pathl (11) & -0.19 & 0.11 & 0.00 & 0.02 & -0.26 & -0.47 & -0.35 & -0.32 & -0.43 & -0.55 & -0.32 & & \\
Qol G (12) & 0.14 & 0.10 & 0.07 & -0.10 & 0.27 & 0.21 & 0.23 & 0.50 & 0.45 & 0.70 & 0.56 & -0.61 & \\
Qol S & 0.12 & 0.07 & 0.03 & 0.02 & 0.21 & 0.27 & 0.36 & 0.38 & 0.29 & 0.28 & 0.46 & -0.45 & 0.43 \\
\hline
\end{tabular}

Note. Living= Independent living; Friend= Friendship; Self= Sense of self; Sat med= Satisfaction with medical care; Sat act= Satisfaction with daily activities; Ps path= Psychopathology; Education=Educational level; Act level= Activity level; Qol G= General quality of life; Qol S= the quality of life aspect labelled Security. 

Table 3. Statistics for the measurement and path models.

\begin{tabular}{l|rrrrrrrrrrr}
\hline & Chi-square & $d f$ & $p$ & RMSEA & GFI & AGFI & CFI & NFI & PNFI & MAIC & $E C V I$ \\
\hline MeasQOL1 & 54.23 & 27 & 0.001 & 0.089 & 0.92 & 0.86 & 0.93 & 0.87 & 0.66 & 109.32 & 0.82 \\
MeasQOL2 & 30 & 23 & 0.150 & 0.043 & 0.95 & 0.91 & 0.98 & 0.93 & 0.60 & 90.61 & 0.68 \\
Model1 & 237.39 & 29 & 0.001 & 0.29 & 0.65 & 0.34 & 0.70 & 0.68 & 0.44 & 425.65 & 3.20 \\
Model2 & 243.43 & 34 & 0.001 & 0.23 & 0.65 & 0.48 & 0.71 & 0.67 & 0.55 & 413.91 & 3.11 \\
Model3 & 6.19 & 9 & 0.001 & 0.00 & 0.99 & 0.94 & 1.00 & 0.99 & 0.20 & 118.06 & 0.83 \\
Model4 & 127.26 & 28 & 0.001 & 0.17 & 0.83 & 0.66 & 0.86 & 0.83 & 0.52 & 212.17 & 1.60 \\
Model5 & 58.61 & 22 & 0.001 & 0.13 & 0.91 & 0.53 & 0.95 & 0.92 & 0.45 & 150.78 & 1.16 \\
Model6 & 9.09 & 16 & 0.001 & 0.00 & 0.99 & 0.95 & 1.0 & 0.99 & 0.35 & 106.96 & 0.78 \\
\hline
\end{tabular}




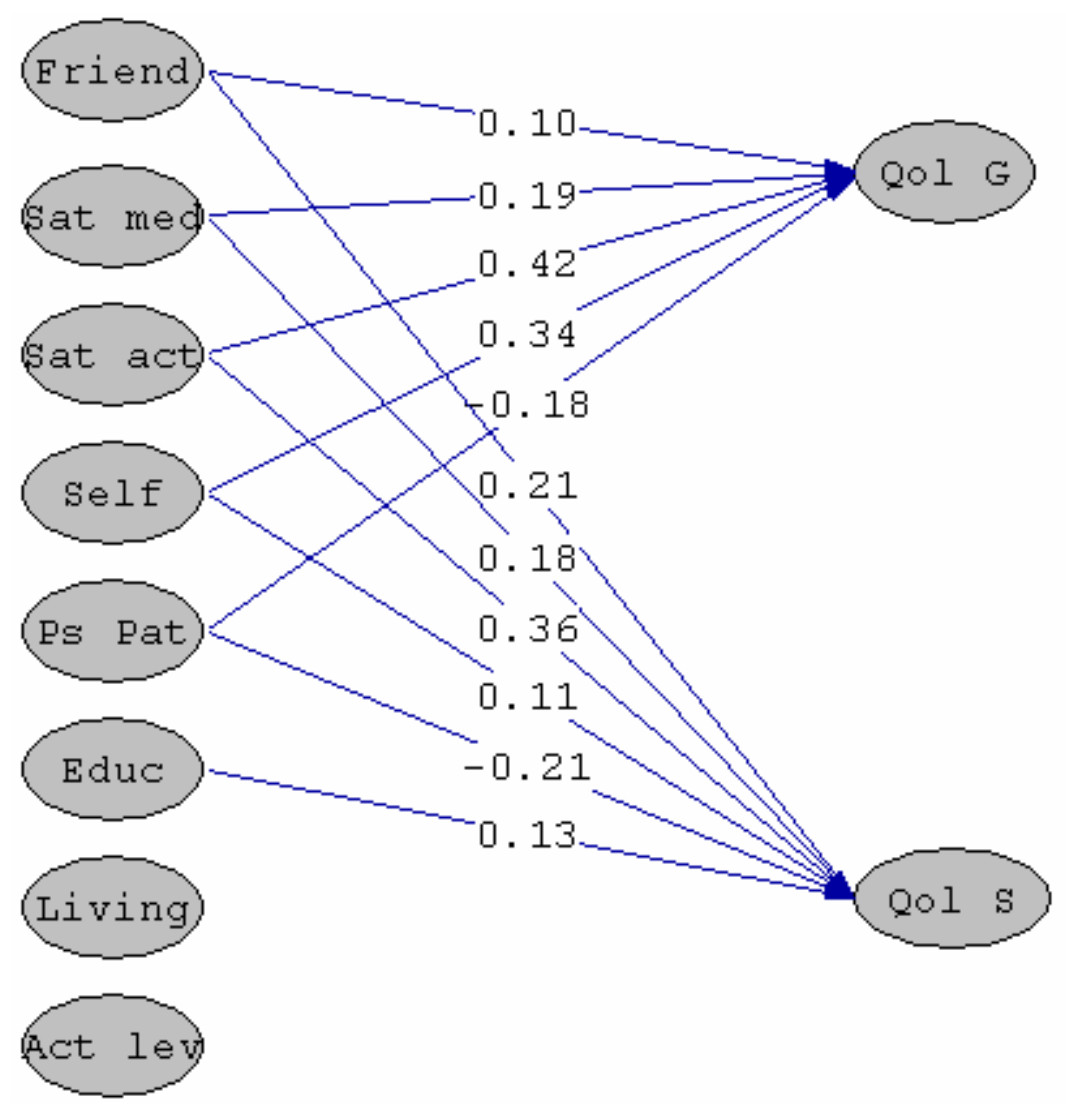

Note . Living= Independent living; Friend= Friendship; Self= Sense of self; Sat med= Satisfaction with medical care; Sat act= Satisfaction with daily activities; Ps path= Psychopathology; Education= Educational level; Act level= Activity level; Qol G= General quality of life; Qol S= the quality of life aspect labelled Security. 


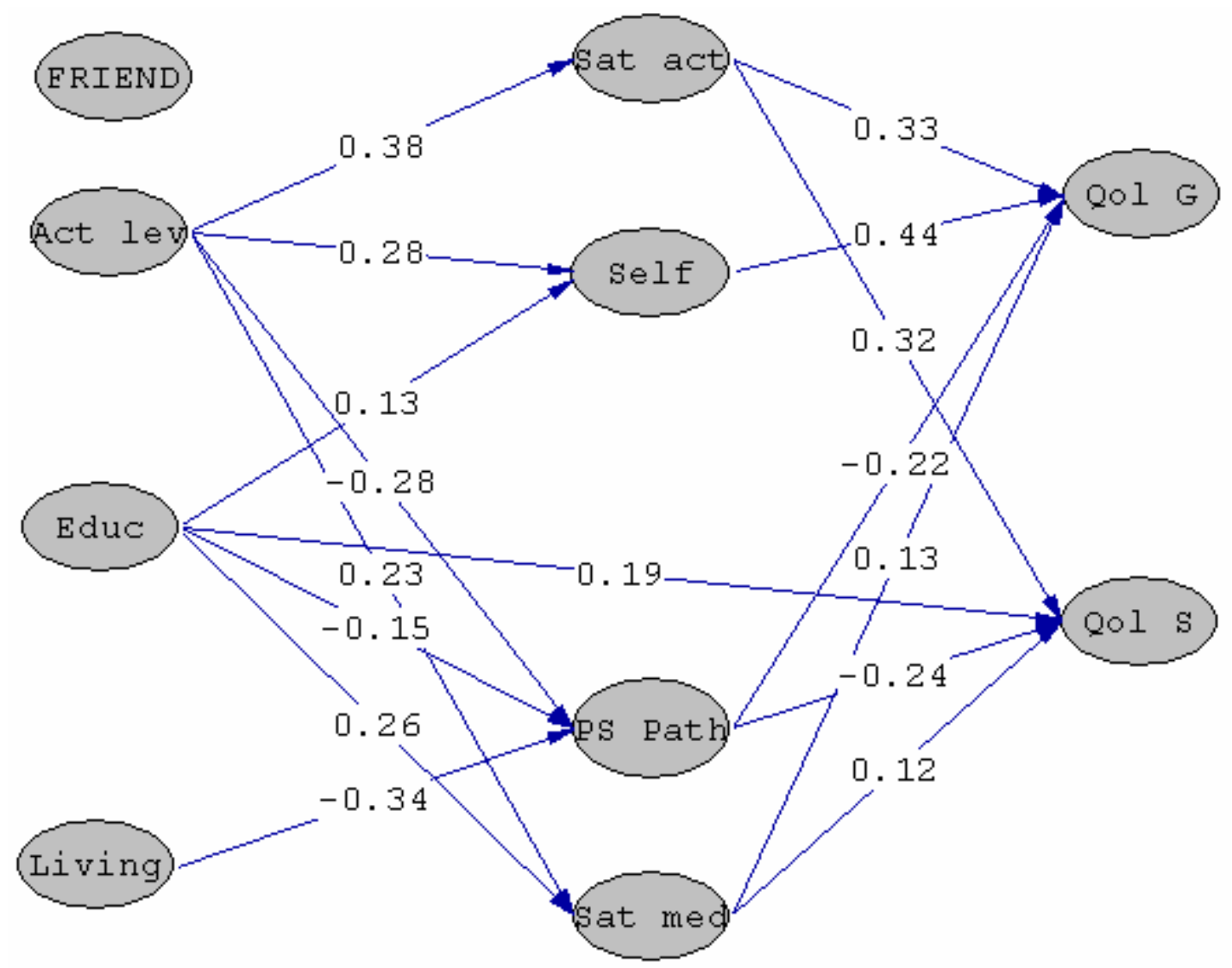

Note. Living= Independent living; Friend= Friendship; Self $=$ Sense of self; Sat med= Satisfaction with medical care; Sat act= Satisfaction with daily activities; Ps path= Psychopathology; Education= Educational level; Act level= Activity level; Qol G= General quality of life; Qol S= the quality of life aspect labelled Security. 